\title{
Viral membrane fusion: is glycoprotein $G$ of rhabdoviruses a representative of a new class of viral fusion proteins?
}

A.T. Da Poian,

F.A. Carneiro and F. Stauffer
Programa de Biologia Molecular e Biotecnologia, Instituto de Bioquímica Médica, Universidade Federal do Rio de Janeiro, Rio de Janeiro, RJ, Brasil

\section{Correspondence}

A.T. Da Poian

Instituto de Bioquímica Médica UFRJ

21941-590 Rio de Janeiro, RJ

Brasil

E-mail: dapoian@bioqmed.ufr..br

Presented at the XI Congresso Brasileiro de Biologia Celular, Campinas, SP, Brazil, July 15-18, 2004.

Research supported by CNPq, Centro Argentino-Brasileiro de Biotecnologia (CABBIO) and FAPERJ.

Received July 16, 2004 Accepted February 10, 2005

\begin{abstract}
Enveloped viruses always gain entry into the cytoplasm by fusion of their lipid envelope with a cell membrane. Some enveloped viruses fuse directly with the host cell plasma membrane after virus binding to the cell receptor. Other enveloped viruses enter the cells by the endocytic pathway, and fusion depends on the acidification of the endosomal compartment. In both cases, virus-induced membrane fusion is triggered by conformational changes in viral envelope glycoproteins. Two different classes of viral fusion proteins have been described on the basis of their molecular architecture. Several structural data permitted the elucidation of the mechanisms of membrane fusion mediated by class I and class II fusion proteins. In this article, we review a number of results obtained by our laboratory and by others that suggest that the mechanisms involved in rhabdovirus fusion are different from those used by the two well-studied classes of viral glycoproteins. We focus our discussion on the electrostatic nature of virus binding and interaction with membranes, especially through phosphatidylserine, and on the reversibility of the conformational changes of the rhabdovirus glycoprotein involved in fusion. Taken together, these data suggest the existence of a third class of fusion proteins and support the idea that new insights should emerge from studies of membrane fusion mediated by the $\mathrm{G}$ protein of rhabdoviruses. In particular, the elucidation of the three-dimensional structure of the $\mathrm{G}$ protein or even of the fusion peptide at different $\mathrm{pH}$ 's might provide valuable information for understanding the fusion mechanism of this new class of fusion proteins.
\end{abstract}

\section{Introduction to viral-induced membrane fusion}

The plasma membrane of eukaryotic cells serves as a barrier against invading parasites and viruses. To infect a cell, viruses must be capable of transporting their genome and accessory proteins into the host cell, bypass-
Key words

- Membrane fusion

- Fusion proteins

- Rhabdovirus

- G protein

- Glycoprotein 
Figure 1. Entry of enveloped virus into the host cells. First, the virion attaches to the cell surface through the interaction between a viral envelope protein and a receptor molecule, leading to (a) $\mathrm{pH}$-independent fusion between viral envelope and host cell plasma membrane or to (b) endocytosis of the virus particle, followed by fusion of the endosomal membrane with the viral envelope at low pH. After membrane fusion, the viral genome is released into the cytosol to be replicated, transcribed and translated. In both cases, membrane fusion is mediated by specific viral surface glycoproteins, which undergo structural reorganization to be converted from the nonfusogenic to the fusogenic conformation. brane with viral envelope, after virus particle internalization by receptor-mediated endocytosis $(1,2)$. In both cases, membrane fusion is mediated by specific viral surface glycoproteins which undergo structural reorganization to be converted from the nonfusogenic to the fusogenic conformation.

The membrane of some enveloped viruses, such as paramyxoviruses, retroviruses or herpesviruses, fuses directly with the host cell plasma membrane after virus binding to their cell receptor. The best-characterized fusion mechanism that occurs at the plasma membrane level is that mediated by HIV gp120/gp41 glycoproteins (3). Interaction between gp120, the cellular CD4 molecule and a co-receptor protein leads to the insertion of gp41 hydrophobic fusion peptide into the plasma membrane. gp41 forms a trimeric coiled coil containing two interacting $\alpha$-helical peptides that acquire a six-helical bundle

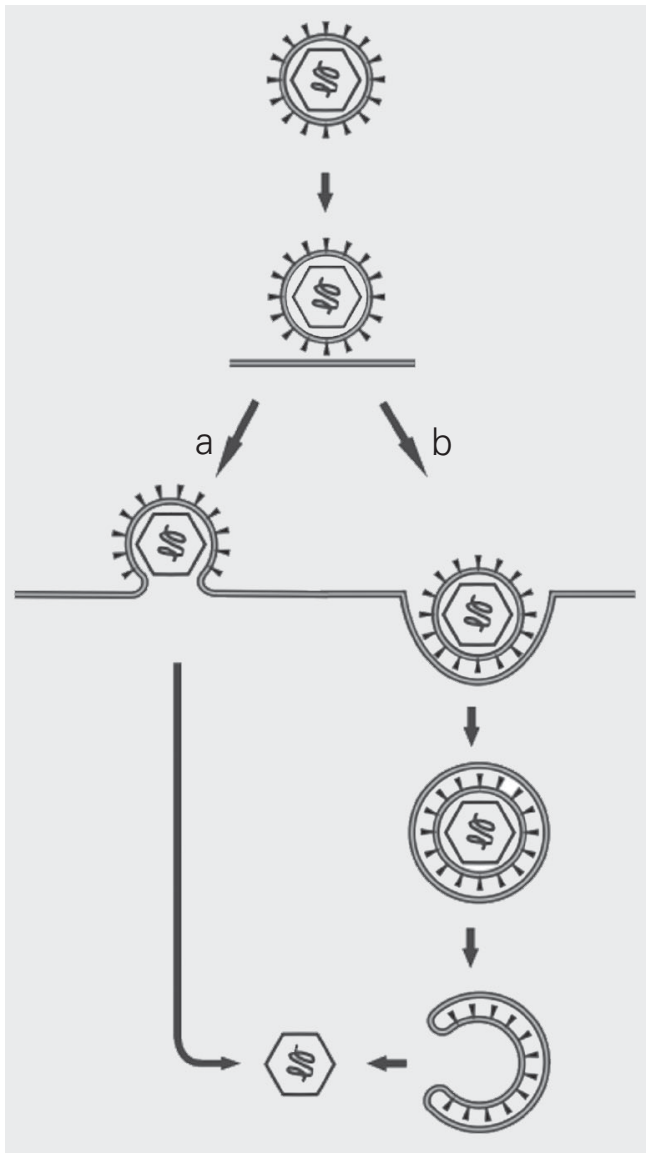

structure (4).

Other enveloped viruses such as orthomyxoviruses, alphaviruses or rhabdoviruses enter the cells by the endocytic pathway, and fusion depends on the acidification of the endosomal compartment. Fusion at the endosome level is triggered by conformational changes in viral glycoproteins induced by the low $\mathrm{pH}$ of this cellular compartment. The best-studied low $\mathrm{pH}$-activated viral fusion protein is the influenza virus glycoprotein hemagglutinin (HA). The X-ray structure of influenza virus HA was determined at both neutral and fusogenic $\mathrm{pH}$ (5). The conformational changes observed suggest that the hydrophobic fusion peptide moves to the tip of the molecule and is delivered toward the target membrane. The conformational transition occurs in a narrow $\mathrm{pH}$ range, corresponding to the optimal $\mathrm{pH}$ of fusion, in which the protein acquires the ability to interact with detergent micelles and lipid vesicles. This interaction leads to the insertion of the fusion peptide into the membrane, where a pore is formed.

\section{Viral fusion proteins}

The viral fusion glycoproteins share a number of common features: a) they are type I integral membrane proteins presenting a large ectodomain, a single transmembrane sequence and a small C-terminal end inside the viral membrane; b) they contain an $\mathrm{N}$ terminal signal sequence that is cleaved after directing the protein at the endoplasmic reticulum; c) they contain N-linked carbohydrates; d) they form oligomers and occur at high density in the viral membrane; e) they contain a specific segment involved in membrane fusion known as the fusion peptide.

Based on their molecular architecture, two classes of viral fusion proteins have been described. The main features that characterize the proteins of each class are summarized in Table 1. The HA from influenza 
virus is the prototype of class I fusion proteins, which also include glycoproteins from myxo- and paramyxoviruses, retroviruses and filoviruses (5). These proteins form trimeric spikes of two-chain monomers generated by the cleavage of a precursor protein. The $\mathrm{C}$ terminal end is anchored to the viral membrane and the N-terminal created by the precursor cleavage consists of a hydrophobic segment containing approximately 20 amino acids, which corresponds to the fusion peptide. After binding to a cellular receptor or on exposure to low $\mathrm{pH}$, the protein assumes an extended conformation and the fusion peptide inserts into the target membrane. The post-fusion conformation is a hairpinlike structure in which the N-terminal central coiled coil is surrounded by a sheath of antiparallel chains, joining the fusion peptide and the membrane anchor at the same end (6).

Class II includes the E protein from flavivirus and E1 protein from alphaviruses (7). They have three domains that fold largely on $\beta$-sheets and are arranged in a continuous protein lattice formed by dimers (8-10). The fusion peptide is an internal loop between two $\beta$-strands, which is buried in the dimer interface. The class II fusion proteins are not proteolytically cleaved during virus maturation, but are associated with another viral membrane protein whose cleavage is required for fusion. The post-fusion structure of $\mathrm{E}$ protein of dengue virus (11), tick-borne encephalitis virus (12), and E1 protein of Semliki Forest virus (13) has been recently determined, revealing a surprising convergence of the class I and class II fusion mechanisms (14). The acidic $\mathrm{pH}$ of the endosome induces a disassembly of envelope protein dimers, which rearrange in trimers with the fusion peptide loops clustered at one end of an elongated molecule.

Despite their structural differences, both class I and class II fusion proteins are synthesized in a metastable conformation, and it is believed that the irreversible transition to the post-fusion state provides the energy for membrane fusion. However, several results suggest that there is at least one example of viral fusion protein that catalyzes fusion through a completely different mechanism. This is the case for the rhabdovirus fusion glycoprotein.

\section{The rhabdoviruses}

The viruses that belong to the Rhabdoviridae family are widely distributed in nature and their hosts range from vertebrate and invertebrate animals to many species of plants. The rhabdoviruses that infect mam-

Table 1. Characteristics of viral fusion protein classes.

\begin{tabular}{lll}
\hline & Class I & Class II \\
\hline Oligomeric structure & Trimer & Dimer \\
Secondary structure & Predominantly $\alpha$-helix & Predominantly ß-sheet \\
Fusion peptide & N-terminal & Internal \\
Proteolysis during maturation & Fusion protein & Associated membrane protein \\
Virus families & Retroviruses (HIV, SIV, MoLV, HTLV-1) & Alphaviruses (SFV) \\
& Orthomyxoviruses (influenza) & Flaviviruses (dengue, TBE) \\
& Paramyxoviruses (Sendai, SV5, HRSV) & \\
\hline
\end{tabular}

HIV = human immunodeficiency virus; SIV = simian immunodeficiency virus; MoLV = Moloney murine leukemia virus; HTLV-1 = human T-cell leukemia virus-1; SV5 = simian virus 5; HRSV = human respiratory syncytial virus; SFV = Semliki Forest virus; TBE $=$ tick-borne encephalitis virus 
mals have been classified into two genera: the Lyssavirus (rabies and rabies-like viruses) and the Vesiculovirus (whose name is derived from vesicular stomatitis virus (VSV), the prototype of the genus). Other rhabdoviruses include those that infect fish, arthropods and plants. All rhabdoviruses present a bullet-shaped structure measuring $200 \times 70 \mathrm{~nm}$ that is formed by two major components: the nucleocapsid and the envelope. The nucleocapsid contains a negative single-stranded genomic RNA that is tightly packed by the nucleocapsid protein $\mathrm{N}$. The viral RNA-dependent RNA polymerase, composed of $\mathrm{L}$ and $\mathrm{P}$ proteins, is also associated with the nucleocapsid core. The envelope is a lipid bilayer derived from the host cell containing approximately 400 trimeric transmembrane spikes consisting of the single viral glycoprotein G. The matrix protein is localized inside the viral envelope between the membrane and the nucleocapsid.

\section{Entry of rhabdoviruses into host cells}

The rhabdoviruses enter the cell by receptor-mediated endocytosis followed by low-pH-induced membrane fusion. Both the cell recognition and the fusion reaction are mediated by the surface glycoprotein G (1519).

\section{Structural features of rhabdovirus $\mathrm{G}$ protein}

Rhabdoviruses possess a unique glycoprotein in their envelope, which presents a highly conserved structure (20). G proteins contain about 500 amino acids including a signal peptide, two sites of glycosylation, two acylated sites, and a hydrophilic cytoplasmic C-terminal tail. Rabies virus and VSV G proteins are organized as trimers anchored to the viral membrane via a single transmembrane sequence close to the C-terminus (21-24). The trimeric structure of VSV $\mathrm{G}$ protein is stabilized at mild acidic $\mathrm{pH}(22)$ but both rabies and VSV G protein trimers seem to be less stable than the other trimeric viral glycoproteins $(24,25)$.

\section{Rhabdovirus binding to the cell surface}

The receptors for the attachment of rhabdoviruses have been difficult to identify because of the generally broad host range of these viruses. It has been hypothesized that the nicotinic acetylcholine receptor is the receptor for rabies virus $(26,27)$, but recent evidence indicates that other proteins can also act as receptors for this virus $(28,29)$.

Binding to phospholipids seems to be important for rhabdovirus infection. Phospholipids from cellular membranes inhibit attachment and infection of rabies virus and VSV (30-32). Indeed, the VSV host range extends from nearly all mammals to insects, suggesting that the receptor for this virus is a widely distributed molecule. A high affinity, saturable binding site has been described for VSV on Vero cells (30). The binding was inhibited by a membrane extract, which was resistant to protease, neuraminidase and heating, and was also inactivated by treatment with phospholipase C (33). These findings, taken together with the observation that only phosphatidylserine (PS) among various purified lipids was able to inhibit VSV binding, led the authors to suggest that PS could participate in the cellular binding site for VSV (33). The components of biological membranes are asymmetrically distributed between the membrane surfaces and PS is highly segregated to the inner leaflet of plasma membranes, suggesting that G protein-PS interactions were a very improbable event. However, recent findings showing that the binding of a fragment of a salmonid rhabdovirus $G$ protein to model membranes induces PS translocation from the inner to the outer leaflet of the membrane (34) indicate that rhabdovirus binding to PS could have more physiological relevance than expected. Another possibility is that other negatively charged molecules such as glycosami- 
noglycans and gangliosides could act as the physiological binding site for VSV. The results obtained by Mastromarino et al. (31) using erythrocytes at acidic $\mathrm{pH}$ showed that, besides PS, phosphatidylinositol and GM3 ganglioside also inhibited VSV attachment to cells. Removal of the charged groups from these negatively charged molecules greatly reduced their inhibitory activities, suggesting an important role of electrostatic interactions during cell recognition by VSV.

We found direct evidence that VSV interacts very strongly with membranes containing negatively charged phospholipids at neutral $\mathrm{pH}$. Isothermal titration calorimetry experiments have shown that VSV binding to liposomes is an enthalpically driven process, suggesting that electrostatic interactions are important for membrane recognition (35). Using atomic force microscopy we determined the forces of VSV G protein binding to lipid films of different composition $(35,36)$. We measured forces as strong as $2 \mathrm{nN}$ after virus interaction with a PScontaining lipid film, whereas no interaction between the virus and membranes composed of neutral phospholipids was observed (35).
Using other negatively charged phospholipids, we found that although $\mathrm{G}$ protein can bind to phosphatidylglycerol and cardiolipin, binding to PS seems to be much stronger (36), suggesting that membrane recognition by VSV occurs through a specific interaction between $\mathrm{G}$ protein positively charged amino acids and the negative charges of PS on the membrane surface (Figure 2A).

Viral hemorrhagic septicemia virus (VHSV), a fish rhabdovirus that infects salmonids, also binds PS (37). The PS-binding site of the VHSV G protein has been mapped using pepscan and solid-phase phospholipid binding assays. The major PS-binding regions were located between residues 79 and 113 of VHSV G protein. This sequence presents contiguous heptad repeats followed by a short segment containing positively charged amino acids, and led to the design of a peptide named $\mathrm{p} 2$ (from residues 82 to 109), which contains both the heptad repeat region and two arginine residues (Figure 2B). Antibodies against this peptide were able to inhibit both PS binding to VHSV and virus-induced cell to cell fusion, suggesting a direct participation of $\mathrm{p} 2$ peptide in virus

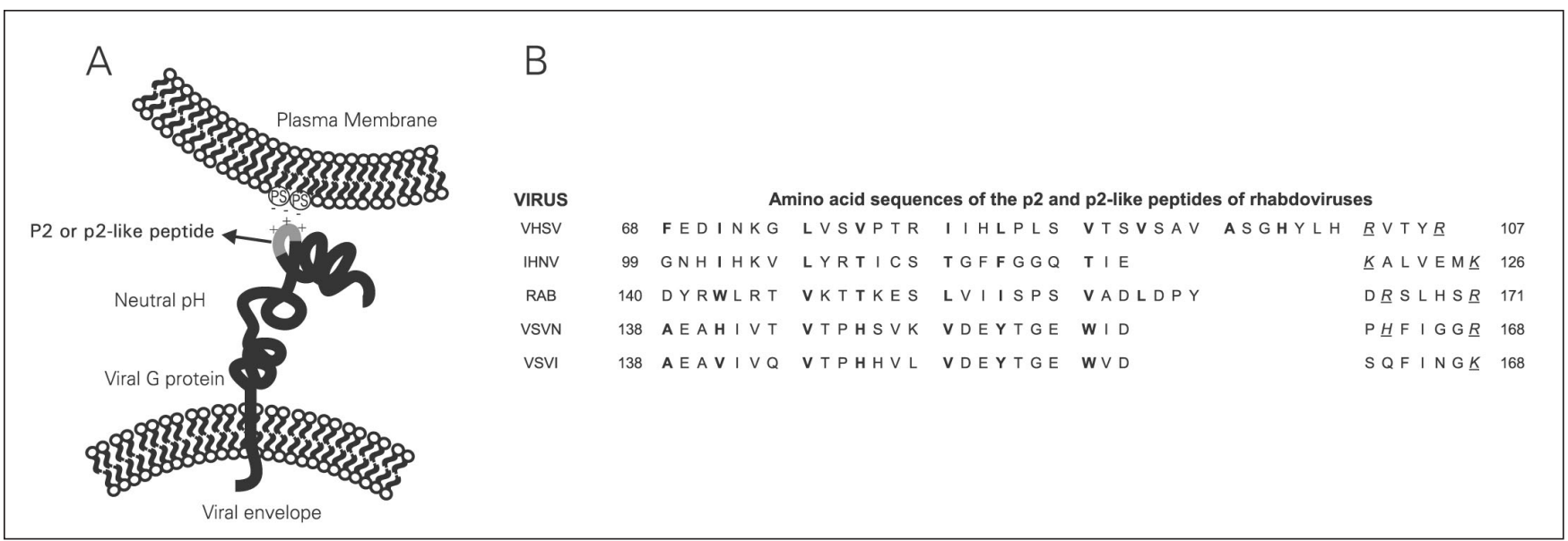

Figure 2. Binding of the $\mathrm{G}$ protein of rhabdoviruses to the cell surface. $A$, Schematic representation of the interaction between viral $\mathrm{G}$ protein and phosphatidylserine (PS) in the plasma membrane, at neutral $\mathrm{pH}$, showing the importance of electrostatic interactions for the binding. A specific sequence of $G$ protein directly involved in the binding to the negatively charged phospholipid PS is shown in gray. This sequence presents similar structural features in the G protein of all rhabdoviruses and is named p2 or p2-like peptide (20). B, PS-binding segments of rhabdovirus G protein contain heptad repeats followed by a short segment containing at least one positively charged residue (20). The heptads are separated by a space for better visualization. The numbers shown on the left or right correspond to the first and the last amino acid residue in each sequence, respectively. VHSV = viral hemorrhagic septicemia virus; IHNV = infectious hematopoietic necrosis virus RB-1 strain; RAB = rabies B19 strain; VSVN = vesicular stomatitis virus New Jersey strain; VSVI = vesicular stomatitis virus Indiana strain. 
entry (37). p2-like regions have been found in other rhabdoviruses (38). These sequences contain heptad repeats followed by a short sequence of 5 to 7 amino acids with at least one positively charged residue, and show a high degree of sequence conservation within the same genus, but no sequence similarity among the different genera (Figure 2B).

p2-like peptides from rabies (residues 140 to 164 ), VSV (residues 134 to 161) and infectious hematopoietic necrosis virus, another fish rhabdovirus (residues 99 to 119), bind PS (39). The identification of these phospholipid-binding domains in several rhabdovirus $G$ proteins suggests that PS binding is a common feature of rhabdovirus envelope protein. These observations led us to propose that the binding of VSV to PScontaining membranes occurs through its p2-like peptide (Figure 2A). Positive charges in this segment might participate in electrostatic interactions with the negatively charged phospholipid PS during membrane recognition.

\section{Rhabdovirus-induced membrane fusion}

\section{G protein conformational changes}

The low $\mathrm{pH}$-induced conformational changes of rhabdovirus $\mathrm{G}$ protein were first investigated for VSV. It has been shown that the incubation of the protein at mild acidic $\mathrm{pH}$ leads to an exposure of a hydrophobic region (25). Binding of the fluorescent probe bis-ANS revealed that the exposure of hydrophobic domains was maximal at $\mathrm{pH} 6.2$ (40). Between $\mathrm{pH} 6.0$ and 5.6 a dramatic conformational change occurs, which includes loss of secondary and tertiary structures. For rabies virus $\mathrm{G}$ protein, it has been shown that immediately after acidification, at $\mathrm{pH}$ below 6.7, the viruses become more hydrophobic allowing the interaction with membranes (41). This conformation of $G$ protein has been considered to be an acti- vated state that may trigger the first step of membrane fusion. In the absence of membranes, however, the hydrophobic conformation of rabies and VSV G protein leads to its aggregation $(18,40)$. Prolonged incubation at low $\mathrm{pH}$ leads rabies $\mathrm{G}$ protein to an inactive state, which become sensitive to proteases and is antigenically distinct from the native structure (18). For VSV, G trimers are stabilized in the inactive state (22).

\section{Role of phosphatidylserine}

It seems that PS is not only involved in membrane recognition but is also of crucial importance in membrane fusion, especially in the case of VSV. Membrane fusion mediated by VSV G protein reconstituted in lipid vesicles showed a large preference for target membranes containing PS or phosphatidic acid (42). We found that the extent of $\mathrm{pH}$ induced conformational changes of $\mathrm{G}$ protein depends on the number of negative charges in the target membrane and that $G$ protein-mediated membrane fusion may be correlated with the PS content in the vesicles (35). Although our results unequivocally demonstrate the importance of electrostatic interactions for VSV fusion, we cannot exclude the possibility that hydrophobic interactions are also involved in VSV interaction with membranes during fusion.

\section{Role of histidines}

We have shown that VSV-induced fusion depends on a dramatic structure reorganization of $\mathrm{G}$ protein, which occurs within a very narrow $\mathrm{pH}$ range, between 5.8 and 6.2 (40). This indicates that the protonation of a small number of ionizable groups is required for $G$ protein structural changes, suggesting that the protonation of the imidazole ring of histidyl residue(s) $(\mathrm{pK}=6.0)$ is involved in $\mathrm{G}$ protein conformational changes required for fusion. In addition, we have found that VSV binding to membranes as well as the 
fusion reaction itself were highly dependent on electrostatic interactions between negative charges on the membrane surface and positively charged amino acids in $\mathrm{G}$ protein at the fusion $\mathrm{pH}$ (35), and His becomes positively charged after protonation (Figure $3)$. For rabies virus, Roche and Gaudin (43), showed that the $\mathrm{pK}$ of the transition from the native to the fusion-inactive state of $\mathrm{G}$ protein is 6.65 , also suggesting that His residues are involved. To investigate the role of His protonation in VSV fusion, we modified these residues using diethyl pyrocarbonate, a compound that reacts with a nitrogen atom of the imidazole ring of His forming $\mathrm{N}$ carbethoxyhistidyl derivatives (44) (Figure $3)$. His modification abolished $\mathrm{pH}$-induced conformational changes on $\mathrm{G}$ protein and also the fusion reaction catalyzed by the virus, suggesting that His protonation drives $\mathrm{G}$ protein interaction with the target membrane at acidic $\mathrm{pH}$ (Figure 3). Mass spectrometry analysis of tryptic fragments of modified $G$ protein, together with the use of synthetic peptides, allowed the identification of His 148 and His 149 of VSV G protein as the putative active residues (44). These His residues are located in the p2-like peptide of VSV, the PS-binding domain first identified in VHSV $G$ protein and found among all rhabdoviruses $(37,39)$.

Further evidence for the involvement of His protonation in rhabdovirus fusion was obtained in the study of the $\mathrm{pH}$-dependent equilibrium between the native and the fusion-inactive states of rabies virus $\mathrm{G}$ protein

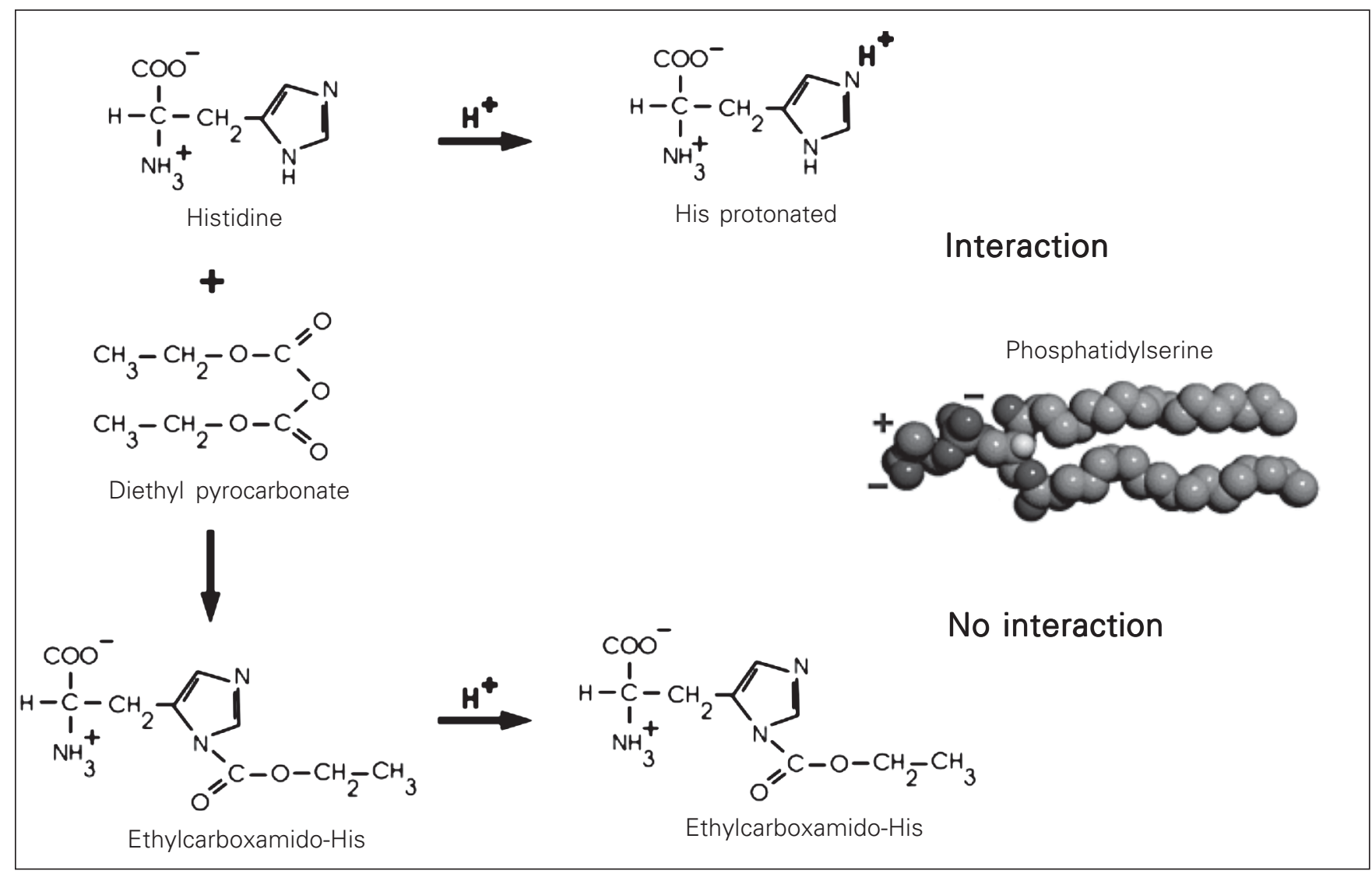

Figure 3. Role of His protonation in rhabdovirus-induced membrane fusion. His modification with diethyl pyrocarbonate (DEPC) abolishes pH-induced conformational changes in $\mathrm{G}$ protein and also the fusion reaction catalyzed by the virus. As demonstrated in this figure, treatment of vesicular stomatitis virus with DEPC impairs His protonation, which seems to be necessary for the interaction between $\mathrm{G}$ protein and the negative charges conferred by phosphatidylserine to the target membrane. 
(43). Based on other studies using natural mutants (45), the authors suggested that the protonation of His 397 was required for the $\mathrm{pH}-\mathrm{dependent} \mathrm{structural} \mathrm{transitions.} \mathrm{Thus,}$ the protonation of this residue together with the protonation of His 148 and 149 would drive the complete conformational changes of $\mathrm{G}$ protein during membrane fusion mediated by the virus.

\section{Determination of the rhabdovirus fusion peptide}

The identification of the amino acid residues essential for membrane fusion mediated by viral glycoproteins might contribute to the elucidation of the molecular mechanisms underlying the fusion event.

Photolabeling studies of rabies and VSV G proteins have shown that interaction of these proteins with membranes strongly increased when the $\mathrm{pH}$ was lowered from 7.0 to 6.0 (46). At the $\mathrm{pH}$ of fusion, the labeling sites are contained in a segment in the ectodomain comprising amino acids 103 to 179 for rabies and 59 to 221 for VSV. In the case of VSV, mutational analysis has shown that substitution of conserved Gly, Pro, or Asp located in the region between amino acids 117 to 137 either abolished the fusion ability of $G$ protein or shifted the optimum $\mathrm{pH}$ of fusion (47-49). Based on these results, the authors proposed that this segment contained VSV G protein internal fusion peptide. However, although this segment is highly conserved among the vesiculoviruses, there is less homology in the corresponding region in rabies glycoprotein (46) and direct evidence that this particular region interacts with the target membrane is still lacking. VSV putative fusion peptide contains no His, and thus it cannot be modified by diethyl pyrocarbonate. In addition, we found that a synthetic peptide corresponding to this segment failed to induce phospholipid vesicle fusion (44). Thus, further investigation is necessary to determine whether the segment between amino acids 117 to 137 of $\mathrm{G}$ protein directly participates in VSV fusion or whether the substitution of its conserved amino acids affects the conformation or the exposure of other membrane-interacting sequence in $\mathrm{G}$ protein.

In contrast, we found that VSV p2-like peptide was as efficient as the whole virus in catalyzing membrane fusion (44). In addition, peptide-induced fusion showed the same requirements as VSV-mediated fusion. It occurs at $\mathrm{pH} 6.0$ but not at $\mathrm{pH} 7.5$, and depends on the presence of PS on the target membrane. The data obtained with $\mathrm{p} 2$ peptide of VHSV also indicate that this peptide might play an active role in fusion: it mediates phospholipid vesicle fusion, lipid mixing, and leakage of liposome contents and inserts itself into liposome membranes (50). These results together suggest that p2-like peptides directly participate in membrane fusion mediated by rhabdoviruses probably through the protonation of their His residues.

Another region of $\mathrm{G}$ protein, encompassing residues 395 to 418 for VSV or containing residues 392 to 396 for rabies virus, has been identified as a segment that affects the fusogenic activity of the protein by influencing the low-pH-induced conformational changes $(45,51)$. In addition, it has also been shown that not only the ectodomain segment but also the membrane anchoring domain is required for VSV fusion activity $(52,53)$.

\section{Reversibility}

It has been suggested that the fusogenic conformation of viral fusion glycoprotein is thermodynamically more stable than the native structure of the protein, which is trapped in a metastable state (54). The native state is prevented from achieving the lower energy fusogenic conformation by a kinetic barrier imposed during the folding and/or maturation. In the case of influenza virus, for example, HA folds within the cell as the fu- 
sion-incompetent precursor, which subsequently undergoes proteolytic cleavage to generate the mature, two-chain native state. This metastability allows the coupling of an energetically expensive membrane-fusion reaction to an energetically favorable conformational change, a fact that could drive the reaction toward complete membrane fusion (54).

The most striking observation with respect to the conformational changes of rhabdovirus $\mathrm{G}$ proteins is the reversibility of the structural transitions, suggesting that metastability is not absolutely required for viral membrane fusion. For rabies virus, it has been demonstrated that $\mathrm{G}$ protein can assume at least three different conformations: the native state detected on the viral surface at neutral $\mathrm{pH}$, the activated hydrophobic state, and the fusion-inactive (I) state (41). These different conformations occur in a $\mathrm{pH}-\mathrm{de}-$ pendent equilibrium and the low-pH triggered conformational changes are completely reversible $(18,41)$. Although the metastability model predicts that any destabilizing reagent will cause the same conformational change and membrane-fusion activity as acidic $\mathrm{pH}$, for $\mathrm{VSV}$, neither heat nor urea induced the conformational changes leading to membrane fusion (55).

An even more interesting observation is that the low $\mathrm{pH}$-induced conformational changes of rhabdovirus fusion proteins are reversible also after the interaction with the membrane $(56,57)$. It has been shown that after the formation of the rabies virus prefusion complex, the lipids organized in this structure can be reversed to two bilayers (56). Photosensitized labeling experiments have shown that VSV binding to membranes and the subsequent interaction at low $\mathrm{pH}$ could be reversed after medium neutralization, suggesting a "velcro"-like attachment of VSV G protein to the target membrane (57). This indicates that $G$ protein interaction with the target membrane during fusion occurs essentially on the membrane surface, and not through the insertion of a protein segment into the lipid bilayer. This hypothesis is in agreement with our results showing the electrostatic nature of $\mathrm{G}$ protein-lipid interactions (35), and suggests a mechanism of membrane destabilization resembling the one promoted by the antimicrobial peptides acting through a carpet model (58).

The increasing knowledge about virusinduced membrane fusion at the molecular level should provide new means to develop antiviral drugs. Indeed, although most of the antiviral drugs have been developed against viral enzymes involved in virus replication, recent studies have demonstrated that the entry events can also serve as a new target to block viral infection $(59,60)$. As an example, the progress in understanding the mechanisms of HIV-1 cell entry into target cells permitted the design of a new class of antiHIV-1 drugs: compounds that act as fusion or entry inhibitors that are currently being evaluated in clinical trials (60). Thus, new insights concerning the fusion mechanisms might be successfully applied to the development of entry inhibitors directed at other viruses. The data presented in this review suggest that the glycoprotein of the rhabdoviruses represents a new class of fusion proteins. The elucidation of the three-dimensional structure of this glycoprotein or even of its fusion peptide should provide valuable information for understanding the fusion mechanism of this new class of fusion proteins. 


\section{References}

1. Hernandez LD, Hoffman LR, Wolfsberg TG \& White JM (1996). Virus-cell and cell-cell fusion. Annual Review of Cell and Developmental Biology, 12: 627-661.

2. Eckert DM \& Kim PS (2001). Mechanisms of viral membrane fusion and its inhibition. Annual Review of Biochemistry, 70: 777-810.

3. Chan DC \& Kim PS (1998). HIV entry and its inhibition. Cell, 93: 681684.

4. Chan DC, Fass D, Berger JM \& Kim PS (1997). Core structure of gp41 from the HIV envelope glycoprotein. Cell, 89: 263-273.

5. Skehel JJ \& Wiley DC (2000). Receptor binding and membrane fusion in virus entry: the influenza hemagglutinin. Annual Review of Biochemistry, 69: 531-569.

6. Skehel JJ \& Wiley DC (1998). Coiled coils in both intracellular vesicle and viral membrane fusion. Cell, 95: 871-874.

7. Heinz FX \& Allison SL (2001). The machinery for flavivirus fusion with host cell membranes. Current Opinion in Microbiology, 4: 450455.

8. Rey FA, Heinz FX, Mandl C, Kunz C \& Harrison SC (1995). The envelope glycoprotein from tick-borne encephalitis virus at $2 \mathrm{~A}$ resolution. Nature, 375: 291-298.

9. Lescar J, Roussel A, Wien MW, Navaza J, Fuller SD, Wengler G, Wengler G \& Rey FA (2001). The fusion glycoprotein shell of Semliki Forest virus: an icosahedral assembly primed for fusogenic activation at endosomal pH. Cell, 105: 137-148.

10. Kuhn RJ, Zhang W, Rossmann MG et al. (2002). Structure of dengue virus: implications for flavivirus organization, maturation, and fusion. Cell, 108: 717-725.

11. Modis Y, Ogata S, Clements D \& Harrison SC (2004). Structure of the dengue virus envelope protein after membrane fusion. Nature, 427: 313-319

12. Bressanelli S, Stiasny K, Allison SL, Stura EA, Duquerroy S, Lescar J, Heinz FX \& Rey FA (2004). Structure of a flavivirus envelope glycoprotein in its low-pH-induced membrane fusion conformation. EMBO Journal, 23: 728-738.

13. Gibbons DL, Vaney MC, Roussel A, Vigouroux A, Reilly B, Lepault J, Kielian M \& Rey FA (2004). Conformational change and proteinprotein interactions of the fusion protein of Semliki Forest virus. Nature, 427: 320-325.

14. Jardetzky TS \& Lamb RA (2004). Virology: a class act. Nature, 427: 307-308.

15. Matlin KS, Reggio H, Helenius A \& Simons K (1982). Pathway of vesicular stomatitis virus entry leading to infection. Journal of Molecular Biology, 156: 609-631.

16. Superti F, Derer M \& Tsiang H (1984). Mechanism of rabies virus entry into CER cells. Journal of General Virology, 65: 781-789.

17. Wunner WH, Reagan KJ \& Koprowski H (1984). Characterization of saturable binding sites for rabies virus. Journal of Virology, 50: 691697.

18. Gaudin Y, Tuffereau C, Segretain D, Knossow M \& Flamand A (1991). Reversible conformational changes and fusion activity of rabies virus glycoprotein. Journal of Virology, 65: 4853-4859.

19. Da Poian AT, Gomes AMO \& Coelho-Sampaio T (1998). Kinetics of intracellular viral disassembly and processing probed by Bodipy fluorescence dequenching. Journal of Virological Methods, 70: 4558

20. Coll JM (1995). The glycoprotein G of rhabdoviruses. Archives of Virology, 140: 827-851.

21. Kreis TE \& Lodish HF (1986). Oligomerization is essential for transport of vesicular stomatitis viral glycoprotein to the cell surface.
Cell, 46: 929-937.

22. Doms RW, Keller DS, Helenius A \& Balch WE (1987). Role for adenosine triphosphate in regulating the assembly and transport of vesicular stomatitis virus $\mathrm{G}$ protein trimers. Journal of Cell Biology, 105: 1957-1969.

23. Whitt MA, Buonocore L, Prehaud C \& Rose JK (1991). Membrane fusion activity, oligomerization, and assembly of the rabies virus glycoprotein. Virology, 185: 681-688.

24. Gaudin $Y$, Ruigrok RW, Tuffereau $C$, Knossow $M$ \& Flamand $A$ (1992). Rabies virus glycoprotein is a trimer. Virology, 187: 627-632.

25. Crimmins DL, Mehard WB \& Schlesinger S (1983). Physical properties of a soluble form of the glycoprotein of vesicular stomatitis virus at neutral and acidic pH. Biochemistry, 22: 5790-5796.

26. Lentz TL, Burrage TG, Smith AL, Crick J \& Tignor GH (1982). Is the acetylcholine receptor a rabies virus receptor? Science, 215: 182184.

27. Gastka M, Horvath J \& Lentz TL (1996). Rabies virus binding to the nicotinic acetylcholine receptor alpha subunit demonstrated by virus overlay protein binding assay. Journal of General Virology, 77: 2437-2440.

28. Reagan KJ \& Wunner WH (1985). Rabies virus interaction with various cell lines is independent of the acetylcholine receptor. Archives of Virology, 84: 277-282.

29. Thoulouze MI, Lafage M, Schachner M, Hartmann U, Cremer H \& Lafon M (1998). The neural cell adhesion molecule is a receptor for rabies virus. Journal of Virology, 72: 7181-7190.

30. Schlegel R, Willingham MC \& Pastan IH (1982). Saturable binding sites for vesicular stomatitis virus on the surface of Vero cells. Journal of Virology, 43: 871-875.

31. Mastromarino P, Conti C, Goldoni P, Hauttecoeur B \& Orsi N (1987). Characterization of membrane components of the erythrocyte involved in vesicular stomatitis virus attachment and fusion at acidic pH. Journal of General Virology, 68: 2359-2369.

32. Conti C, Mastromarino P, Ciuffarella MG \& Orsi N (1988). Characterization of rat brain cellular membrane components acting as receptors for vesicular stomatitis virus. Brief report. Archives of Virology, 99: 261-269.

33. Schlegel R, Tralka TS, Willingham MC \& Pastan I (1983). Inhibition of VSV binding and infectivity by phosphatidylserine: is phosphatidylserine a VSV-binding site? Cell, 32: 639-646.

34. Estepa AM, Rocha Al, Mas $V$ et al. (2001). A protein $G$ fragment from the salmonid viral hemorrhagic septicemia rhabdovirus induces cell-to-cell fusion and membrane phosphatidylserine translocation at low pH. Journal of Biological Chemistry, 276: 4626846275.

35. Carneiro FA, Bianconi ML, Weissmuller G, Stauffer F \& Da Poian AT (2002). Membrane recognition by vesicular stomatitis virus involves enthalpy-driven protein-lipid interactions. Journal of Virology, 76: 3756-3764.

36. Weissmüller G, Moraes MB, Prazeres GMP et al. (2003). Multiple application of atomic force microscopy in biology. Acta Microscopica, 12: 29-33

37. Estepa AM \& Coll JM (1996). Pepscan mapping and fusion-related properties of the major phosphatidylserine-binding domain of the glycoprotein of viral hemorrhagic septicemia virus, a salmonid rhabdovirus. Virology, 216: 60-70.

38. Coll JM (1995). Heptad-repeat sequences in the glycoprotein of rhabdoviruses. Virus Genes, 10: 107-114.

39. Coll JM (1997). Synthetic peptides from the heptad repeats of the 
glycoproteins of rabies, vesicular stomatitis and fish rhabdoviruses bind phosphatidylserine. Archives of Virology, 142: 2089-2097.

40. Carneiro FA, Ferradosa AS \& Da Poian AT (2001). Low pH-induced conformational changes in vesicular stomatitis virus glycoprotein involve dramatic structure reorganization. Journal of Biological Chemistry, 276: 67-72.

41. Gaudin Y, Ruigrok RW, Knossow M \& Flamand A (1993). Low-pH conformational changes of rabies virus glycoprotein and their role in membrane fusion. Journal of Virology, 67: 1365-1372.

42. Eidelman O, Schlegel R, Tralka TS \& Blumenthal R (1984). pHdependent fusion induced by vesicular stomatitis virus glycoprotein reconstituted into phospholipid vesicles. Journal of Biological Chemistry, 259: 4622-4628.

43. Roche S \& Gaudin Y (2002). Characterization of the equilibrium between the native and the fusion-inactive conformation of the rabies virus glycoprotein indicates that the fusion complex is made of several trimers. Virology, 297: 128-135.

44. Carneiro FA, Stauffer F, Lima CS, Juliano MA, Juliano L \& Da Poian AT (2003). Membrane fusion induced by vesicular stomatitis virus depends on histidine protonation. Journal of Biological Chemistry, 278: 13789-13794.

45. Gaudin Y, Raux H, Flamand A \& Ruigrok RW (1996). Identification of amino acids controlling the low-pH-induced conformational change of rabies virus glycoprotein. Journal of Virology, 70: 7371-7378.

46. Durrer P, Gaudin Y, Ruigrok WH, Graf R \& Brunner J (1995). Photolabeling identifies a putative fusion domain in the envelope glycoprotein of rabies and vesicular stomatitis viruses. Journal of Biological Chemistry, 270: 17575-17581.

47. Li Y, Drone C, Sat E \& Ghosh HP (1993). Mutational analysis of the vesicular stomatitis virus glycoprotein $G$ for membrane fusion domains. Journal of Virology, 67: 4070-4077.

48. Zhang L \& Ghosh HP (1994). Characterization of the putative fusogenic domain in vesicular stomatitis virus glycoprotein G. Journal of Virology, 68: 2186-2193.

49. Fredericksen BL \& Whitt M (1995). Vesicular stomatitis virus glycoprotein mutations that affect membrane fusion activity and abolish virus infectivity. Journal of Virology, 69: 1435-1443.

50. Nuñez E, Fernandez AM, Estepa A, Gonzalez-Ros JM, Gavilanes F \&
Coll JM (1998). Phospholipid interactions of a peptide from the fusion-related domain of the glycoprotein of VHSV, a fish rhabdovirus. Virology, 243: 322-330.

51. Shokralla S, He Y, Wanas E \& Ghosh HP (1998). Mutations in a carboxy-terminal region of vesicular stomatitis virus glycoprotein $\mathrm{G}$ that affect membrane fusion activity. Virology, 242: 39-50.

52. Odell D, Wanas E, Yan J \& Ghosh HP (1997). Influence of membrane anchoring and cytoplasmic domains on the fusogenic activity of vesicular stomatitis virus glycoprotein G. Journal of Virology, 71: 7996-8000.

53. Cleverley DZ \& Lenard J (1998). The transmembrane domain in vira fusion: essential role for a conserved glycine residue in vesicular stomatitis virus $\mathrm{G}$ protein. Proceedings of the National Academy of Sciences, USA, 95: 3425-3430.

54. Carr CM, Chaudhry C \& Kim PS (1997). Influenza hemagglutinin is spring-loaded by a metastable native conformation. Proceedings of the National Academy of Sciences, USA, 94: 14306-14313.

55. Yao Y, Ghosh K, Epand RF, Epand RM \& Ghosh HP (2003). Membrane fusion activity of vesicular stomatitis virus glycoprotein $G$ is induced by low pH but not by heat or denaturant. Virology, 310: 319332.

56. Gaudin Y (2000). Rabies virus-induced membrane fusion pathway. Journal of Cell Biology, 150: 601-611.

57. Pak CC, Puri A \& Blumenthal R (1997). Conformational changes and fusion activity of vesicular stomatitis virus glycoprotein: [125|]iodonaphthyl azide photolabeling studies in biological membranes. Biochemistry, 36: 8890-8896.

58. Shai $Y$ (1999). Mechanism of the binding, insertion and destabilization of phospholipid bilayer membranes by alpha-helical antimicrobial and cell non-selective membrane-lytic peptides. Biochimica et Biophysica Acta, 1462: 55-70.

59. Stoll V, Stewart KD, Maring CJ et al. (2003). Influenza neuraminidase inhibitors: structure-based design of a novel inhibitor series. Biochemistry, 42: 718-727.

60. Moore JP \& Doms RW (2003). The entry of entry inhibitors: a fusion of science and medicine. Proceedings of the National Academy of Sciences, USA, 100: 10598-10602. 